\title{
IMPULSIVE NONLOCAL NONLINEAR PARABOLIC DIFFERENTIAL PROBLEMS ${ }^{1}$
}

\author{
LUDWIK BYSZEWSKI \\ Cracow University of Technology \\ Institute of Mathematics \\ Cracow 31-155, POLAND
}

\begin{abstract}
The aim of the paper is to prove a theorem about a weak impulsive nonlinear parabolic differential inequality together with weak impulsive nonlocal nonlinear inequalities. A weak maximum principle for an impulsive nonlinear parabolic differential inequality together with weak impulsive nonlocal nonlinear inequalities and an uniqueness criterion for the existence of the classical solution of an impulsive nonlocal nonlinear parabolic differential problem are obtained as a consequence of the theorem about the weak impulsive nonlinear parabolic differential inequality together with weak impulsive nonlocal nonlinear inequalities.
\end{abstract}

Key words: Impulsive parabolic problems, nonlocal conditions, arbitrary parabolic sets, differential inequalities, uniqueness criterion.

AMS (MOS) subject classifications: 35R45, 35B50, 35K20, $35 \mathrm{~K} 60,35 \mathrm{~A} 05,35 \mathrm{~K} 99$.

\section{INTRODUCTION}

In this paper we prove a theorem about a weak impulsive nonlinear parabolic differential inequality together with weak impulsive nonlocal nonlinear inequalities. The impulsive inequality, studied here, is of the form

$$
u_{t}-f\left(t, x, u, u_{x}, u_{x x}\right) \leq v_{t}-f\left(t, x, v, v_{x}, v_{x x}\right),
$$

where $(t, x) \in\left({ }^{s} \bigcup^{-1} D \cap\left[\left(t_{i}, t_{i+1}\right) \times \mathbb{R}^{n}\right]\right) \cup\left(D \cap\left[\left(t_{s}, t_{0}+T\right] \times \mathbb{R}^{n}\right]\right), \quad s$ is a fixed natural number, $\stackrel{i=0}{D}$ is one of two relatively arbitrary sets more general than the cylindrical domain $\left(t_{0}, t_{0}+T\right] \times D_{0} \subset \mathbb{R}^{n+1}$ and

$$
t_{0}<t_{1}<t_{2}<\ldots<t_{s}<t_{0}+T \text {. }
$$

\footnotetext{
${ }^{1}$ Received: March, 1993. Revised: June, 1993.
} 
The impulsive nonlocal inequalities, considered here, are of the form

$$
u\left(t_{i}, x\right)+\sum_{j \in I_{i}^{*}} h_{i, j}(x) G_{i, j}(x, u) \leq v\left(t_{i}, x\right)+\sum_{j \in I_{i}^{*}} h_{i, j}(x) G_{i, j}(x, v),
$$

where $I_{i}^{*} \quad(i=0,1, \ldots, s)$ are subsets of countable sets $I_{i}(i=0,1, \ldots, s)$, respectively, $\quad t_{i}<T_{i, 2 j-1}<T_{i, 2 j}<t_{i+1} \quad\left(j \in I_{i}^{*}, i=0,1, \ldots, s-1\right), \quad t_{s}<T_{s, 2 j-1}$ $<T_{s, 2 j} \leq t_{0}+T\left(j \in I_{s}^{*}\right), h_{i, j}: S_{t_{i}} \rightarrow(-\infty, 0]$ and $G_{i, j}: S_{t_{i}} \times C\left(\left[T_{i, 2 j-1}, T_{i, 2 j}\right] \times S_{t_{i}}\right)$ $\rightarrow \mathbb{R}\left(j \in I_{i}^{*}, i=0,1, \ldots, s\right)$ are given functions satisfying some assumptions and

$$
S_{t_{i}}:=\operatorname{int}\left\{x \in \mathbb{R}^{n}:\left(t_{i}, x\right) \in D\right\} \quad(i=0,1, \ldots, s) .
$$

As a consequence of the theorem about the weak impulsive nonlinear parabolic differential inequality together with weak impulsive nonlocal nonlinear inequalities we obtain a weak maximum principle for an impulsive nonlinear parabolic differential inequality together with weak impulsive nonlocal nonlinear inequalities and an uniqueness criterion for the existence of the classical solution of an impulsive nonlocal nonlinear parabolic differential problem.

Many processes in the theories of heat conduction and diffusion are characterized by the fact that at certain moments $t_{1}, t_{2}, \ldots, t_{s}$ of time they experience changes of temperatures of a heated substance or changes of amounts of a diffused substance. Moreover, for many above processes we know the relations between the temperatures of the heated substance and we know the relations between the amounts of the diffused substance at the points $t_{i}, T_{i, 2 j-1}$, $T_{i, 2 j}\left(j \in I_{i}^{*}, i=0,1, \ldots, s-1\right)$ and $t_{s}, T_{s, 2 j-1}, T_{s, 2 j}\left(j \in I_{s}^{*}\right)$. Consequently, it is natural to assume that these changes act in the form of impulses at the points $t_{1}, t_{2}, \ldots, t_{s}$ and that the following impulsive nonlocal conditions are considered

$$
u\left(t_{i}, x\right)+\sum_{j \in I_{i}^{*}} h_{i, j}(x) G_{i, j}(x, u)=\phi_{i}(x) \text { for } x \in S_{t_{i}} \quad(i=0,1, \ldots, s),
$$

where $\phi_{i}(i=0,1, \ldots, s)$ are given real functions defined on $S_{t_{i}}(i=0,1, \ldots, s)$, respectively.

It is easy to see from (1.1) that these conditions are more general than the standard initial conditions. Moreover, if

$$
G_{i, j}(x, u):=u\left(T_{i, 2 j}, x\right) \text { for } x \in S_{t_{i}} \quad\left(j \in I_{i}^{*}, i=0,1, \ldots, s\right)
$$




$$
G_{i, j}(x, u):=\frac{1}{T_{i, 2 j}-T_{i, 2 j-1}} \int_{T_{i, 2 j-1}}^{T_{i, 2 j}} u(\tau, x) d \tau \text { for } x \in S_{t_{i}} \quad\left(j \in I_{i}^{*}, i-0,1, \ldots, s\right)
$$

then conditions (1.1) are reduced to the impulsive periodic conditions and to the impulsive antiperiodic conditions, or to the impulsive average periodic conditions and to the impulsive average antiperiodic conditions for suitable functions $h_{i, j}$ $\left(j \in I_{i}^{*}, i=0,1, \ldots, s\right)$.

To obtain physical interpretations of the impulsive nonlocal problems considered in the paper it is enough to join the physical interpretations of the nonlocal problems and of the impulsive problems. For this purpose, compare papers [2] and [3], where physical interpretations of the nonlocal problems and of the impulsive problems were given separately.

The paper is a continuation and a generalization of papers [1]-[3]. Moreover, the paper generalizes some theorems from [4] and [5]. To prove the main result of this paper a strong maximum principle from the author publication [1] is used.

\section{PRELIMINARIES}

The notation, definitions and assumptions given in this section are valid throughout the paper.

Let $t_{0}$ be real finite number, $0<T<\infty$ and let $x=\left(x_{1}, \ldots, x_{n}\right) \in \mathbb{R}^{n}$. A bounded or unbounded set $D$ contained in $\left(t_{0}, t_{0}+T\right] \times \mathbb{R}^{n}$ and satisfying the conditions:

(a) The projection of the interior of $D$ on the $t$-axis is the interval $\left(t_{0}, t_{0}+T\right)$.

(b) For any $(\tilde{t}, \tilde{x}) \in D$ there exists a positive number $r$ such that

$$
\left\{(t, x):(t-\tilde{t})^{2}+\sum_{i=1}^{n}\left(x_{i}-\tilde{x}_{i}\right)^{2}<r, t<\tilde{t}\right\} \subset D
$$

is said to be a set of type $(P)$.

For any $t \in\left[t_{0}, t_{0}+T\right]$ we define the following sets:

$$
S_{t}:=\left\{\begin{array}{cc}
\operatorname{int}\left\{x \in \mathbb{R}^{n}:\left(t_{0}, x\right) \in \bar{D}\right\} & \text { for } t=t_{0} \\
\left\{x \in \mathbb{R}^{n}:(t, x) \in D\right\} & \text { for } t \neq t_{0}
\end{array}\right.
$$


and

$$
\sigma_{t}:=\left\{\begin{array}{cc}
\operatorname{int}\left[\bar{D} \cap\left(\left\{t_{0}\right\} \times \mathbb{R}^{n}\right)\right] & \text { for } t=t_{0}, \\
D \cap\left(\{t\} \times \mathbb{R}^{n}\right) & \text { for } t \neq t_{0}
\end{array}\right.
$$

It is easy to see, by condition (b) of the definition of a set of type $(P)$, that $S_{t}$ and $\sigma_{t}$, where $t \in\left[t_{0}, t_{0}+T\right]$, are open sets in $\mathbb{R}^{n}$ and $\mathbb{R}^{n+1}$, respectively.

By $s$ we denote a fixed number belonging to $\mathbb{N}$ or $\mathbb{N}_{0}$.

Let $t_{1}, t_{2}, \ldots, t_{s} \quad(s \in \mathbb{N})$ be given real numbers such that

$$
t_{0}<t_{1}<t_{2}<\ldots<t_{s}<t_{0}+T \quad(s \in \mathbb{N}) .
$$

We introduce the following sets:

$$
\begin{gathered}
D_{i}=D \cap\left[\left(t_{i}, t_{i+1}\right) \times \mathbb{R}^{n}\right] \quad(i=0,1, \ldots, s-1 ; s \in \mathbb{N}), \\
D_{s}:=D \cap\left[\left(t_{s}, t_{0}+T\right] \times \mathbb{R}^{n}\right] \quad\left(s \in \mathbb{N}_{0}\right), \\
D(s):=\bigcup_{i=0}^{s} D_{i} \quad\left(s \in \mathbb{N}_{0}\right)
\end{gathered}
$$

and

$$
\sigma(s):=\left\{\begin{array}{cc}
\emptyset & \text { if } s=0, \\
\bigcup_{i=1}^{s} \bar{\sigma}_{t_{i}} & \text { if } s \in \mathbb{N} .
\end{array}\right.
$$

It is easy to see that $D(0)=D_{0}=D$.

Let

where

$$
\Gamma(s):=\bigcup_{i=0}^{s} \Gamma_{i} \quad\left(s \in \mathbb{N}_{0}\right)
$$

$$
\begin{gathered}
\Gamma_{i}:=\Gamma \cap\left(\left[t_{i}, t_{i+1}\right) \times \mathbb{R}^{n}\right) \quad(i=0,1, \ldots, s-1 ; s \in \mathbb{N}), \\
\Gamma_{s}:=\Gamma \cap\left(\left[t_{s}, t_{0}+T\right] \times \mathbb{R}^{n}\right) \quad\left(s \in \mathbb{N}_{0}\right)
\end{gathered}
$$

and

$$
\Gamma:=(\bar{D} \backslash D) \backslash \sigma_{t_{0}}
$$

For an arbitrary fixed point $(\tilde{t}, \tilde{x}) \in D$ we denote by $S^{-}(\tilde{t}, \tilde{x})$ the set of points $(t, x) \in D$ that can be joined with $(\tilde{t}, \tilde{x})$ by a polygonal line contained in $D$ along which the $t$-coordinate is weakly increasing from $(t, x)$ to $(\tilde{t}, \tilde{x})$.

By $P C(\bar{D})$ we denote the space of functions 


$$
w: \bar{D} \ni(t, x) \rightarrow w(t, x) \in \mathbb{R}
$$

such that $w$ is continuous in $\bar{D} \backslash \sigma(s) \quad\left(s \in \mathbb{N}_{0}\right)$, the finite limits $w\left(t_{i}^{-}, x\right), w\left(t_{i}^{+}, x\right)$ $(i=1, \ldots, s)$ exist for all admissible $x \in \mathbb{R}^{n}$ if $s \in \mathbb{N}$ and $w\left(t_{i}, x\right):=w\left(t_{i}^{+}, x\right)$ $(i=1, \ldots, s)$ for all admissible $x \in \mathbb{R}^{n}$ if $s \in \mathbb{N}$.

We say that $w \in P C^{1,2}(\bar{D})$ if $w \in P C(\bar{D})$ and $w_{t}, w_{x}, w_{x x}=\left[w_{x_{j} x_{k}}\right]_{n \times n}$ are continuous in $D(s)\left(s \in \mathbb{N}_{0}\right)$.

The symbol $M_{n \times n}(\mathbb{R})$ is used for the space of real square symmetric matrices $r=\left[r_{j k}\right]_{n \times n}$.

By $f$ we denote a function

$$
f: D(s) \times \mathbb{R} \times \mathbb{R}^{n} \times M_{n \times n}(\mathbb{R}) \ni(t, x, z, q, r) \rightarrow f(t, x, z, q, r) \in \mathbb{R} \quad\left(s \in \mathbb{N}_{0}\right),
$$

where $q=\left(q_{1}, \ldots, q_{n}\right)$ and $r=\left[r_{j k}\right]_{n \times n}$, and by $P$ we denote an operator given by the formula

$$
(P w)(t, x):=w_{t}(t, x)-f\left(t, x, w(t, x), w_{x}(t, x), w_{x x}(t, x)\right), w \in P C^{1,2}(\bar{D}),(t, x) \in D .
$$

Functions $u$ and $v$ belonging to $P C^{1,2}(\bar{D})$ are called solutions of the differential inequality

$$
(P u)(t, x) \leq(P v)(t, x), \quad(t, x) \in D(s) \quad\left(s \in \mathbb{N}_{0}\right)
$$

in $D(s) \quad\left(s \in \mathbb{N}_{0}\right)$, if they satisfy (2.1) for all $(t, x) \in D(s) \quad\left(s \in \mathbb{N}_{0}\right)$.

The function $f$ is said to be uniformly parabolic in a subset $E \subset D(s)$ $\left(s \in \mathbb{N}_{0}\right)$ with respect to a function $w \in P C^{1,2}(\bar{D})$ if there exists a contact $\kappa>0$ (depending on $E$ ) such that for any two matrices $\tilde{r}=\left[\tilde{r}_{j k}\right], \widehat{r}=\left[\widehat{r}_{j k}\right] \in M_{n \times n}(\mathbb{R})$ and for $(t, x) \in E$ we have

$$
\begin{gathered}
\tilde{r} \leq \widehat{r} \Rightarrow f\left(t, x, w(t, x), w_{x}(t, x), \widehat{r}\right)-f\left(t, x, w(t, x), w_{x}(t, x), \tilde{r}\right) \\
\geq \kappa \sum_{i=1}^{n}\left(\widehat{r}_{i i}-\tilde{r}_{i i}\right),
\end{gathered}
$$

where $\tilde{r} \leq \widehat{r}$ means that $\sum_{j, k=1}^{n}\left(\widetilde{r}_{j k}-\widehat{r}_{j k}\right) \lambda_{j} \lambda_{k} \leq 0$ for every $\left(\lambda_{1}, \ldots, \lambda_{n}\right) \in \mathbb{R}^{n}$.

If (2.2) is satisfied with $\kappa=0$ for $\tilde{r}=w_{x x}(t, x)$ and $\widehat{r}=w_{x x}(t, x)+r$, where $r \geq 0$, then $f$ is called parabolic with respect to $w$ in $E$. 
Let us define the sets:

$$
\varphi_{i}=\bigcup_{j \in I_{i}}\left(\sigma_{T_{i, 2 j-1}} \cup \sigma_{T_{i, 2 j}}\right) \quad\left(i=0,1, \ldots, s ; s \in \mathbb{N}_{0}\right),
$$

where $I_{i}\left(i=0,1, \ldots, s ; s \in \mathbb{N}_{0}\right)$ are countable sets of all mutually different natural numbers such that

$\left(i_{1}\right) \quad t_{i}<T_{i, 2 j-1}<T_{i, 2 j}<t_{i+1} \quad$ for $j \in I_{i} \quad$ and $\quad T_{i, 2 j-1} \neq T_{i, 2 k-1}$, $T_{i, 2 j} \neq T_{i, 2 k}$

for $j, k \in I_{i}, j \neq k \quad(i=0,1, \ldots, s-1 ; s \in \mathbb{N})$,

(i, $\tau_{i}:=\inf _{j \in I_{i}} T_{i, 2 j-1}>t_{i}$ and $T_{i}:=\sup _{j \in I_{i}} T_{i, 2 j}<t_{i+1}$ if $\quad \operatorname{card} I_{i}=\aleph_{0}$ $(i=0,1, \ldots, s-1 ; s \in \mathbb{N})$,

$\left(i_{3}\right) \quad S_{t} \supset S_{t_{i}}$ for every $t \in \bigcup_{j \in I_{i}}\left[T_{i, 2 j-1}, T_{i, 2 j}\right] \quad(i=0,1, \ldots, s-1 ; s \in \mathbb{N})$,

$\left(i_{4}\right) \quad S_{t} \supset S_{t_{i}}$ for every $t \in\left[\tau_{i}, T_{i}\right]$ if $\operatorname{card} I_{i}=\aleph_{0} \quad(i=0,1, \ldots, s-1 ; s \in \mathbb{N})$,

$\left(s_{1}\right) \quad t_{s}<T_{s, 2 j-1}<T_{s, 2 j} \leq t_{0}+T$ for $j \in I_{s}$ and $T_{s, 2 j-1} \neq T_{s, 2 k-1}$, $T_{s, 2 j} \neq T_{s, 2 k}$ for $j, k \in I_{s}, j \neq k \quad\left(s \in \mathbb{N}_{0}\right)$,

(s) $\tau_{s}:=\inf _{j \in I_{s}} T_{s, 2 j-1}>t_{s}$ if $\operatorname{cardI} I_{s}=\aleph_{0}\left(s \in \mathbb{N}_{0}\right)$,

(s) $S_{t} \supset S_{t_{s}}$ for every $t \in \bigcup_{j \in I_{s}}\left[T_{s, 2 j-1}, T_{s, 2 j}\right] \quad\left(s \in \mathbb{N}_{0}\right)$,

$\left(s_{4}\right) \quad S_{t} \supset S_{t_{s}}$ for every $t \in\left[\tau_{s}, t_{0}+T\right]$ if $\operatorname{card} I_{s}=\aleph_{0} \quad\left(s \in \mathbb{N}_{0}\right)$.

An unbounded set $D$ of type $(P)$ is called a set of type $\left(P_{I S \Gamma}\right)$ if

$$
\begin{gathered}
\varphi_{i} \neq \emptyset \quad\left(i=0,1, \ldots, s ; s \in \mathbb{N}_{0}\right), \\
\Gamma_{i} \cap \bar{\sigma}_{t_{i}} \neq \emptyset \quad\left(i=0,1, \ldots, s ; \quad s \in \mathbb{N}_{0}\right) .
\end{gathered}
$$

Let $\varphi_{i}^{*} \quad\left(i=0,1, \ldots, s ; \quad s \in \mathbb{N}_{0}\right)$ denote nonempty subsets of $\varphi_{i}$ $\left(i=0,1, \ldots, s ; s \in \mathbb{N}_{0}\right)$, respectively. We define the following sets:

$$
I_{i}^{*}=\left\{j \in I_{i}: \sigma_{T_{i, 2 j-1}} \cup \sigma_{T_{i, 2 j}} \subset \Psi_{i}^{*}\right\} \quad\left(i=0,1, \ldots, s ; s \in \mathbb{N}_{0}\right) .
$$

A bounded set $D$ of type $(P)$ satisfying condition $(a)$ of the definition of a set of type $\left(P_{I S \Gamma}\right)$ is called a set of type $\left(P_{I S B}\right)$.

It is easy to see that if $D$ is a set of type $\left(P_{I S B}\right)$, then $D$ satisfies condition $(b)$ of the definition of a set of type $\left(P_{I S \Gamma}\right)$. Moreover, it is obvious that if $D_{0}$ is a bounded subset $\left[D_{0}\right.$ is an unbounded essential subset] of $\mathbb{R}^{n}$, then $D=\left(t_{0}, t_{0}+T\right] \times D_{0}$ is a set of type $\left(P_{I S B}\right)\left[\left(P_{I S \Gamma}\right)\right.$, respectively $]$. 
Assumption $(G)$ : We say that the functions

$G_{i, j}: S_{t_{i}} \times C\left(\left[T_{i, 2 j-1}, T_{i, 2 j}\right] \times S_{t_{i}}\right) \rightarrow \mathbb{R} \quad\left(j \in I_{i}^{*}, \quad i=0,1, \ldots, s ; s \in \mathbb{N}_{0}\right)$

satisfy Assumption $(G)$ if for every fixed points $\widehat{x}_{i} \in S_{t_{i}} \quad\left(i=0,1, \ldots, s ; s \in \mathbb{N}_{0}\right)$ the inequalities

$$
\begin{gathered}
G_{i, j}\left(\widehat{x}_{i}, u\right)-G_{i, j}\left(\widehat{x}_{i}, v\right) \leq \underset{t \in\left[T_{i, 2 j-1}, T_{i, 2 j}\right]}{\max }\left[u\left(t, \widehat{x}_{i}\right)-v\left(t, \widehat{x}_{i}\right)\right] \\
\left(j \in I_{i}^{*}, i=0,1, \ldots, s ; s \in \mathbb{N}_{0}\right)
\end{gathered}
$$

are satisfied, where $u, v \in P C(\bar{D})$.

\section{A THEOREM ABOUT A WEAK INEQUALITY}

Now, we shall prove Theorem 3.1 which is the main result of this paper.

Theorem 3.1: Assume that

1. $\quad D$ is a set of type $\left(P_{I S \Gamma}\right)$.

2. The function $f$ is weakly decreasing with respect to $z$. Moreover, there exists a positive constant $L$ such that the inequality

$$
\begin{gathered}
f(t, x, z, q, r)-f(t, x, \tilde{z}, \tilde{q}, \tilde{r}) \\
\leq L\left(|z-\tilde{z}|+|x| \sum_{j=1}^{n}\left|q_{j}-\tilde{q}_{j}\right|+|x|_{j, k=1}^{2} \sum_{j k}^{n}\left|r_{j k}-\tilde{r}_{j k}\right|\right)
\end{gathered}
$$

is satisfied for all $(t, x) \in D(s)\left(s \in \mathbb{N}_{0}\right), z, \tilde{z} \in \mathbb{R}, q, \tilde{q} \in \mathbb{R}^{n}, r, \tilde{r} \in M_{n \times n}(\mathbb{R})$.

3. The functions $u$ and $v$ belonging to $P C^{1,2}(\bar{D})$ satisfy the inequalities

$$
u(t, x) \leq v(t, x) \text { for }(t, x) \in \Gamma(s) \quad\left(s \in \mathbb{N}_{0}\right)
$$

and

$$
\begin{gathered}
u\left(t_{i}, x\right)+\sum_{j \in I_{i}^{*}} h_{i, j}(x) G_{i, j}(x, u) \\
\leq v\left(t_{i}, x\right)+\sum_{j \in I_{i}^{*}} h_{i, j}(x) G_{i, j}(x, v) \text { for } x \in S_{t_{i}}\left(i=0,1, \ldots, s ; s \in \mathbb{N}_{0}\right),
\end{gathered}
$$

where $G_{i, j}: S_{t_{i}} \times C\left(\left[T_{i, 2 j-1}, T_{i, 2 j}\right] \times S_{t_{i}}\right) \rightarrow \mathbb{R} \quad\left(j \in I_{i}^{*}, \quad i=0,1, \ldots, s ; \quad s \in \mathbb{N}_{0}\right)$ are given functions satisfying Assumption $(G)$ and $h_{i, j}: S_{t_{i}} \rightarrow(-\infty, 0] \quad\left(j \in I_{i}^{*}\right.$, 
$\left.i=0,1, \ldots s ; s \in \mathbb{N}_{0}\right)$ are given functions such that $-1 \leq \sum_{j \in I_{i}^{*}} h_{i, j}(x) \leq 0$ for $x \in S_{t_{i}}$ $\left(i=0,1, \ldots, s ; \quad s \in \mathbb{N}_{0}\right)$ and, additionally, if card $I_{i}^{*}=\aleph_{0} \quad\left(i=0,1, \ldots, s ; s \in \mathbb{N}_{0}\right)$ then the series $\sum_{j \in I_{i}^{*}} h_{i, j}(x) G_{i, j}(x, u), \sum_{j \in I_{i}^{*}} h_{i, j}(x) G_{i, j}(x, v) \quad\left(i=0,1, \ldots, s ; s \in \mathbb{N}_{0}\right)$ are convergent for $x \in S_{t_{i}}\left(i=0,1, \ldots, s ; s \in \mathbb{N}_{0}\right)$.

4. The maxima of $u-v$ are attained on $\bar{D} \cap\left(\left[t_{i}, t_{i+1}\right) \times \mathbb{R}^{n}\right)$ $(i=0,1, \ldots, s-1 ; s \in \mathbb{N})$ and on $\bar{D}_{s}\left(s \in \mathbb{N}_{0}\right)$. Moreover

and

$$
M_{i}:=\max _{(t, x) \in \bar{D} \cap\left(\left[t_{i}, t_{i+1}\right) \times \mathbb{R}^{n}\right)}[u(t, x)-v(t, x)] \quad(i=0,1, \ldots, s-1 ; s \in \mathbb{N})
$$

$$
M_{s}:=\max _{(t, x) \in \bar{D}_{s}}[u(t, x)-v(t, x)] \quad\left(s \in \mathbb{N}_{0}\right)
$$

5. $\quad f$ is parabolic with respect to $u$ in $D(s)\left(s \in \mathbb{N}_{0}\right)$ and uniformly parabolic with respect to $v+M_{i}\left(i=0,1, \ldots, s ; s \in \mathbb{N}_{0}\right)$ in any compact subset of $D_{i}\left(i=0,1, \ldots, s ; s \in \mathbb{N}_{0}\right)$, respectively.

6. $\quad u$ and $v$ are solutions of the differential inequality (2.1) in $D(s)$ $\left(s \in \mathbb{N}_{0}\right)$.

Then

$$
u(t, x) \leq v(t, x) \quad \text { for }(t, x) \in \bar{D}
$$

Proof: To prove Theorem 3.1 we shall consider two cases: (i) $s=0$ and (ii) $s \geq 1$. For this purpose assume that $s=0$ and suppose that

$$
u(\widehat{t}, \widehat{x})>v(\widehat{t}, \widehat{x})
$$

where $(\widehat{t}, \widehat{x})$ is a point belonging to $\bar{D}$. From assumption 4 and from (3.4), there exists

$$
\left(t^{*}, x^{*}\right) \in \bar{D}
$$

such that

$$
M_{0}=u\left(t^{*}, x^{*}\right)-v\left(t^{*}, x^{*}\right)=\max _{(t, x) \in \bar{D}}[u(t, x)-v(t, x)]>0
$$

Inequalities (3.1) and (3.6) imply that

$$
\left(t^{*}, x^{*}\right) \notin \Gamma
$$


Assume, so, that $\left(t^{*}, x^{*}\right) \in D$. Consequently, by assumptions 6,2 and by formula (3.6), the following conditions hold:

$$
\left\{\begin{array}{c}
(P u)(t, x)-\left(P\left(v+M_{0}\right)\right)(t, x) \\
\leq(P u)(t, x)-(P v)(t, x) \leq 0 \text { for }(t, x) \in D \\
u(t, x) \leq v(t, x)+M_{0} \text { for }(t, x) \in \bar{D} \\
u\left(t^{*}, x^{*}\right)=v\left(t^{*}, x^{*}\right)+M_{0} .
\end{array}\right.
$$

Applying the strong maximum principle from [1] to (3.8) we obtain, by assumptions $1,2,5$, that

$$
u(t, x)=v(t, x)+M_{0} \text { for }(t, x) \in S^{-}\left(t^{*}, x^{*}\right) .
$$

Since $u \in C(\bar{D})$ if $s=0$ and since $M_{0}>0$ then (3.1) contradicts to (3.9). Consequently,

$$
\left(t^{*}, x^{*}\right) \notin D \text {. }
$$

Formulas (3.5), (3.7) and (3.10) imply that

$$
\left(t^{*}, x^{*}\right) \in \sigma_{t_{0}}
$$

From the assumption that $u \in C(\bar{D})$, for every $j \in I_{0}^{*}$ it follows that there is $\widetilde{T}_{0, j} \in\left[T_{0,2 j-1}, T_{0,2 j}\right]$ such that

$$
u\left(\widetilde{T}_{0, j}, x^{*}\right)-v\left(\tilde{T}_{0, j}, x^{*}\right)=\underset{t \in\left[T_{0,2 j-1}, T_{0,2 j}\right]}{\max }\left[u\left(t, x^{*}\right)-v\left(t, x^{*}\right)\right]
$$

Consider now two possible cases:
(A) $\sum_{j \in I_{0}^{*}} h_{0, j}(x)=0, \quad x \in S_{t_{0}}$;
(B) $-1 \leq \sum_{j \in I_{0}^{*}} h_{0, j}(x)<0, \quad x \in S_{t_{0}}$.

In case (A) condition (3.11) leads to a contradiction of (3.2) with (3.6).

In case (B) we shall study two possible cases:

(a) $I_{0}^{*}$ is a finite set, i.e., without loss of generality, there is a number $p \in \mathbb{N}$ such that $I_{0}^{*}=\{1,2, \ldots, p\}$.

(b) $\operatorname{card} I_{0}^{*}=\aleph_{0}$.

First, we shall consider case (a). And so, by (3.2), by Assumption ( $G$ ) and by the inequality

$$
u\left(t, x^{*}\right)-v\left(t, x^{*}\right)<u\left(t_{0}, x^{*}\right)-v\left(t_{0}, x^{*}\right) \text { for } t \in \bigcup_{j=1}^{p}\left[T_{0,2 j-1}, T_{0,2 j}\right]
$$


being a consequence of (3.6), (3.11) and of $(a)\left(s_{1}\right),(a)\left(s_{3}\right)$ of the definition of a set of type $\left(P_{I S \Gamma}\right)$, we have

$$
\begin{gathered}
0 \geq\left[u\left(t_{0}, x^{*}\right)+\sum_{j=1}^{p} h_{0, j}\left(x^{*}\right) G_{0, j}\left(x^{*}, u\right)\right] \\
-\left[v\left(t_{0}, x^{*}\right)+\sum_{j=1}^{p} h_{0, j}\left(x^{*}\right) G_{0, j}\left(x^{*}, v\right)\right] \\
=\left[u\left(t_{0}, x^{*}\right)-v\left(t_{0}, x^{*}\right)\right]+\sum_{j=1}^{p} h_{0, j}\left(x^{*}\right)\left[G_{0, j}\left(x^{*}, u\right)-G_{0, j}\left(x^{*}, v\right)\right] \\
\geq\left[u\left(t_{0}, x^{*}\right)-v\left(t_{0}, x^{*}\right)\right] \cdot\left[1+\sum_{j=1}^{p} h_{0, j}\left(x^{*}\right)\right] .
\end{gathered}
$$

Hence

$$
u\left(t_{0}, x^{*}\right) \leq v\left(t_{0}, x^{*}\right) \text { if } 1+\sum_{j=1}^{p} h_{0, j}\left(x^{*}\right)>0 .
$$

Then, from (3.11), we obtain a contradiction of (3.13) with (3.6). Assume now that

$$
\sum_{j=1}^{p} h_{0, j}\left(x^{*}\right)=-1
$$

Since there exists a number $l \in\{1, \ldots, p\}$ such that

$$
u\left(\widetilde{T}_{0, l}, x^{*}\right)-v\left(\tilde{T}_{0, l}, x^{*}\right)=\max _{j=1, \ldots, p}\left[u\left(\widetilde{T}_{0, j}, x^{*}\right)-v\left(\tilde{T}_{0, j}, x^{*}\right)\right]
$$

then we obtain, by (3.14), (3.15), (3.12), by Assumption $(G)$ and by (3.2), that

$$
\begin{gathered}
{\left[u\left(t_{0}, x^{*}\right)-v\left(t_{0}, x^{*}\right)\right]-\left[u\left(\widetilde{T}_{0, l}, x^{*}\right)-v\left(\widetilde{T}_{0, l}, x^{*}\right)\right]} \\
=\left[u\left(t_{0}, x^{*}\right)-v\left(t_{0}, x^{*}\right)\right]+\sum_{j=1}^{p} h_{0, j}\left(x^{*}\right)\left[u\left(\widetilde{T}_{0, l}, x^{*}\right)-v\left(\widetilde{T}_{0, l}, x^{*}\right)\right] \\
\leq\left[u\left(t_{0}, x^{*}\right)-v\left(t_{0}, x^{*}\right)\right]+\sum_{j=1}^{p} h_{0, j}\left(x^{*}\right)\left[u\left(\widetilde{T}_{0, j}, x^{*}\right)-v\left(\widetilde{T}_{0, j}, x^{*}\right)\right] \\
\leq\left[u\left(t_{0}, x^{*}\right)-v\left(t_{0}, x^{*}\right)\right]+\sum_{j=1}^{p} h_{0, j}\left(x^{*}\right)\left[G_{0, j}\left(x^{*}, u\right)-G_{0, j}\left(x^{*}, v\right)\right] \leq 0 .
\end{gathered}
$$

Hence$$
u\left(t_{0}, x^{*}\right)-v\left(t_{0}, x^{*}\right) \leq u\left(\tilde{T}_{0, l}, x^{*}\right)-v\left(\tilde{T}_{0, l}, x^{*}\right) \text { if } \sum_{j=1}^{p} h_{0, j}\left(x^{*}\right)=-1 .
$$

Since, by $(a)\left(i_{1}\right)$ of the definition of a set of type $\left(P_{I S \Gamma}\right), \widetilde{T}_{0, l}>t_{0}$, we get, from (3.11) that condition (3.16) contradicts condition (3.6). This completes the proof of inequality (3.3) if $I_{0}^{*}$ is a finite set. 
It remains to investigate case (b). Analogously, as in the proof of (3.3) in case $(a)$, by $(3.2)$ and by the inequality

$$
u\left(t, x^{*}\right)-v\left(t, x^{*}\right)<u\left(t_{0}, x^{*}\right)-v\left(t_{0}, x^{*}\right) \text { for } t \in \bigcup_{j \in I_{0}^{*}}\left[T_{0,2 j-1}, T_{0,2 j}\right],
$$

being a consequence of (3.6), (3.11), and of $(a)\left(s_{1}\right),(a)\left(s_{3}\right)$ of the definition of a set of type $\left(P_{I S \Gamma}\right)$, we have

Hence

$$
\begin{gathered}
0 \geq\left[u\left(t_{0}, x^{*}\right)+\sum_{j \in I_{0}^{*}} h_{0, j}\left(x^{*}\right) G_{0, j}\left(x^{*}, u\right)\right]-\left[v\left(t_{0}, x^{*}\right)+\sum_{j \in I_{0}^{*}} h_{0, j}\left(x^{*}\right) G_{0, j}\left(x^{*}, v\right)\right] \\
=\left[u\left(t_{0}, x^{*}\right)-v\left(t_{0}, x^{*}\right)\right]+\sum_{j \in I_{0}^{*}} h_{0, j}\left(x^{*}\right)\left[G_{0, j}\left(x^{*}, u\right)-G_{0, j}\left(x^{*}, v\right)\right] \\
\geq\left[u\left(t_{0}, x^{*}\right)-v\left(t_{0}, x^{*}\right)\right] \cdot\left[1+\sum_{j \in I_{0}^{*}} h_{0, j}\left(x^{*}\right)\right] .
\end{gathered}
$$

$$
u\left(t_{0}, x^{*}\right) \leq v\left(t_{0}, x^{*}\right) \text { if } 1+\sum_{j \in I_{0}^{*}} h_{0, j}\left(x^{*}\right)>0 .
$$

Then, from (3.11), we obtain a contradiction of (3.17) with (3.6). Assume now that

$$
\sum_{j \in I_{0}^{*}} h_{0, j}\left(x^{*}\right)=-1
$$

and let $\tilde{\tau}_{0}^{*}:=i n f_{j \in I_{0}^{*}} \widetilde{T}_{0, j}$. Since $u \in C(\bar{D})$ if $s=0$ and since, by $(a)\left(s_{4}\right)$ of the definition of a set of type $\left(P_{I S \Gamma}\right), x^{*} \in S_{t}$ for every $t \in\left[\tau_{0}, t_{0}+T\right]$ if $\operatorname{card} I_{0}=\aleph_{0}$, then there exists a number $\widehat{t} \in\left[\widetilde{\tau}_{0}^{*}, t_{0}+T\right]$ such that

$$
u\left(\widehat{t}, x^{*}\right)-v\left(\widehat{t}, x^{*}\right)=\max _{t \in\left[\widetilde{\tau}_{0}^{*}, t_{0}+T\right]}\left[u\left(t, x^{*}\right)-v\left(t, x^{*}\right)\right] .
$$

Consequently, by (3.18), (3.19), (3.12), by Assumption $(G)$ and by (3.2),

$$
\begin{gathered}
{\left[u\left(t_{0}, x^{*}\right)-v\left(t_{0}, x^{*}\right)\right]-\left[u\left(\widehat{t}, x^{*}\right)-v\left(\widehat{t}, x^{*}\right)\right]} \\
=\left[u\left(t_{0}, x^{*}\right)-v\left(t_{0}, x^{*}\right)\right]+\sum_{j \in I_{0}^{*}} h_{0, j}\left(x^{*}\right)\left[u\left(\widehat{t}, x^{*}\right)-v\left(\widehat{t}, x^{*}\right)\right] \\
\leq\left[u\left(t_{0}, x^{*}\right)-v\left(t_{0}, x^{*}\right)\right]+\sum_{j \in I_{0}^{*}} h_{0, j}\left(x^{*}\right)\left[u\left(\widetilde{T}_{0, j}, x^{*}\right)-v\left(\widetilde{T}_{0, j}, x^{*}\right)\right] \\
\leq\left[u\left(t_{0}, x^{*}\right)-v\left(t_{0}, x^{*}\right)\right]+\sum_{j \in I_{0}^{*}} h_{0, j}\left(x^{*}\right)\left[G_{0, j}\left(x^{*}, u\right)-G_{0, j}\left(x^{*}, v\right)\right] \leq 0 .
\end{gathered}
$$

Hence 


$$
u\left(t_{0}, x^{*}\right)-v\left(t_{0}, x^{*}\right) \leq u\left(\widehat{t}, x^{*}\right)-v\left(\widehat{t}, x^{*}\right) \text { if } \sum_{j \in I_{0}^{*}} h_{0, j}\left(x^{*}\right)=-1 .
$$

Since, by $(a)\left(s_{2}\right)$ of the definition of a set of type $\left(P_{I S \Gamma}\right), \widehat{t}>t_{0}$, we get, from (3.11), that condition (3.20) contradicts condition (3.6). This completes the proof of inequality (3.3) for $s=0$.

To prove Theorem 3.1 in case (ii) assume that $s \geq 1$ and consider the following nonlocal parabolic problems:

$$
\left\{\begin{array}{c}
(P u)(t, x) \leq(P v)(t, x) \text { for }(t, x) \in D \cap\left[\left(t_{0}, a_{1}\right] \times \mathbb{R}^{n}\right], \\
u\left(t_{0}, x\right)+\sum_{j \in I_{0}^{*}} h_{0, j}(x) G_{0, j}(x, u) \\
\leq v\left(t_{0}, x\right)+\sum_{j \in I_{0}^{*}} h_{0, j}(x) G_{0, j}(x, v) \text { for } x \in S_{t_{0}}, \\
u(t, x) \leq v(t, x) \text { for }(t, x) \in \Gamma \cap\left(\left[t_{0}, a_{1}\right] \times \mathbb{R}^{n}\right), \\
a_{1} \text { is an arbitrary fixed number such that } t_{0}<T_{0}<a_{1}<t_{1},
\end{array}\right.
$$

$$
\left\{\begin{array}{c}
(P u)(t, x) \leq(P v)(t, x) \text { for }(t, x) \in D \cap\left[\left(t_{i}, a_{i+1}\right] \times \mathbb{R}^{n}\right] \\
(i=1, \ldots, s-1), \\
u\left(t_{i}, x\right)+\sum_{j \in I_{i}^{*}} h_{i, j}(x) G_{i j}(x, u) \\
\leq v\left(t_{i}, x\right)+\sum_{j \in I_{i}^{*}} h_{i, j}(x) G_{i, j}(x, v) \text { for } x \in S_{t_{i}}(i=1, \ldots, s-1), \\
u(t, x) \leq v(t, x) \text { for }(t, x) \in \Gamma \cap\left(\left[t_{i}, a_{i+1}\right] \times \mathbb{R}^{n}\right)(i=1, \ldots, s-1), \\
a_{i+1}(i=1, \ldots, s-1) \text { are arbitrary fixed numbers such that } \\
t_{i}<T_{i}<a_{i+1}<t_{i+1}(i=1, \ldots, s-1),
\end{array}\right.
$$




$$
\left\{\begin{array}{c}
(P u)(t, x) \leq(P v)(t, x) \text { for }(t, x) \in D_{s}, \\
u\left(t_{s}, x\right)+\sum_{j \in I_{s}^{*}} h_{s, j}(x) G_{s, j}(x, u) \\
\leq v\left(t_{s}, x\right)+\sum_{j \in I_{s}^{*}} h_{s, j}(x) G_{s, j}(x, v) \text { for } x \in S_{t_{s}} \\
u(t, x) \leq v(t, x) \text { for }(t, x) \in \Gamma_{s} .
\end{array}\right.
$$

Applying to problems (3.21)-(3.23) the same argument as in case $(i)$ of the proof of Theorem 3.1, we obtain the inequality

$$
\begin{gathered}
u(t, x) \leq v(t, x) \text { for }(t, x) \in\left[\bar{D} \cap\left(\left[t_{0}, a_{1}\right] \times \mathbb{R}^{n}\right)\right] \\
\cup \bigcup_{i=1}^{s-1}\left[\bar{D} \cap\left(\left[t_{i}, a_{i+1}\right] \times \mathbb{R}^{n}\right)\right] \cup\left[\bar{D} \cap\left(\left[t_{s}, t_{0}+T\right] \times \mathbb{R}^{n}\right)\right] .
\end{gathered}
$$

Since $a_{1}, a_{2}, \ldots, a_{s}$ are arbitrary numbers such that

$$
t_{i}<T_{i}<a_{i+1}<t_{i+1} \quad(i=0,1, \ldots, s-1)
$$

and since $u, v \in P C(\bar{D})$ then from (3.24),

$$
\begin{gathered}
u(t, x) \leq v(t, x) \text { for }(t, x) \in\left[\bar{D} \cap\left(\left[t_{0}, t_{1}\right) \times \mathbb{R}^{n}\right)\right] \cup \bigcup_{i=1}^{s}\left[\bar{D} \cap\left(\left[t_{i}, t_{i+1}\right) \times \mathbb{R}^{n}\right)\right] \\
\cup\left[\bar{D} \cap\left(\left[t_{s}, t_{0}+T\right] \times \mathbb{R}^{n}\right)\right]=\bar{D} \cap\left(\left[t_{0}, t_{0}+T\right] \times \mathbb{R}^{n}\right)=\bar{D} .
\end{gathered}
$$

Therefore, the proof of Theorem 3.1 is complete.

Remark 3.1: If function $v$ from Theorem 3.1 is equal to a constant function then Theorem 3.1 is reduced to the theorem about a weak maximum principle for an impulsive nonlinear parabolic differential inequality together with weak impulsive nonlocal nonlinear inequalities.

Remark 3.2: $\quad$ Theorem 3.1 can also be formulated for a set $D$ of type $\left(P_{I S B}\right)$. For this purpose it is enough to modify only assumption 4 from Theorem 3.1.

\section{UNIQUENESS CRITERION}

As a consequence of Theorem 3.1 we obtain Theorem 4.1 about an uniqueness criterion for the existence of the classical solution of an impulsive nonlocal nonlinear parabolic differential problem. 
Theorem 4.1: Suppose that assumptions 1, 2 of Theorem 3.1 are satisfied. Then in the class of bounded functions $w$ belonging to $P C^{1,2}(\bar{D})$ and such that for all real constants $C$ the function $f$ is uniformly parabolic with respect to $w+C$ in any compact subset of $D(s) \quad\left(s \in \mathbb{N}_{0}\right)$, there exists at most one function $u$ satisfying the following impulsive nonlocal parabolic problem

$$
\begin{gathered}
(P u)(t, x)=0 \text { for }(t, x) \in D(s) \quad\left(s \in \mathbb{N}_{0}\right), \\
u\left(t_{i}, x\right)+\sum_{j \in I_{i}^{*}} h_{i, j}(x) G_{i, j}(x, u)=\phi_{i}(x) \text { for } x \in S_{t_{i}} \quad\left(i=0,1, \ldots, s ; s \in \mathbb{N}_{0}\right), \\
u(t, x)=\psi_{i}(t, x) \text { for }(t, x) \in \Gamma_{i} \quad\left(i=0,1, \ldots, s ; s \in \mathbb{N}_{0}\right),
\end{gathered}
$$

where $\phi_{i}, \quad \psi_{i} \quad\left(i=0,1, \ldots, s ; s \in \mathbb{N}_{0}\right)$ are given functions defined on $S_{t_{i}}, \Gamma_{i}$ $\left(i=0,1, \ldots, s ; s \in \mathbb{N}_{0}\right)$, respectively, $G_{i, j}: S_{t_{i}} \times C\left(\left[T_{i, 2 j-1}, T_{i, 2 j}\right] \times S_{t_{i}}\right) \rightarrow \mathbb{R} \quad\left(j \in I_{i}^{*}\right.$, $\left.i=0,1, \ldots, s ; \quad s \in \mathbb{N}_{0}\right)$ are given functions satisfying Assumption $(G)$ and $h_{i, j}: S_{t_{i}} \rightarrow(-\infty, 0] \quad\left(j \in I_{i}^{*}, i=0,1, \ldots, s ; s \in \mathbb{N}_{0}\right)$ are given functions such that $-1 \leq \sum_{j \in I_{i}^{*}} h_{i, j}(x) \leq 0$ for $x \in S_{t_{i}} \quad\left(i=0,1, \ldots, s ; s \in \mathbb{N}_{0}\right)$ and, additionally, if $\operatorname{card} I_{i}^{*}=\aleph_{0} \quad\left(i=0,1, \ldots s ; s \in \mathbb{N}_{0}\right)$ then the series $\sum_{j \in I_{i}^{*} h_{i, j}}(x) G_{i, j}(x, w)$ $\left(i=0,1, \ldots, s ; s \in \mathbb{N}_{0}\right)$ are convergent for $x \in S_{t_{i}}\left(i=0,1, \ldots, s ; s \in \mathbb{N}_{0}\right)$.

Remark 4.1: Theorem 4.1 can be also formulated for a set $D$ of type $\left(P_{I S B}\right)$.

\section{REFERENCES}

[1] Byszewski, L., Strong maximum principle for implicit nonlinear parabolic functionaldifferential inequalities in arbitrary domains, Univ. Iagel. Acta Math. 24 (1984), 327339 .

[2] Byszewski, L., Strong maximum principles for parabolic nonlinear problems with nonlocal inequalities together with arbitrary functionals, J. Math. Anal. Appl. 156 (1991), 457-470.

[3] Byszewski, L., Impulsive implicit weak nonlinear parabolic functional-differential inequalities, J. Math. Phys. Sci. 26 (1992), 513-528.

[4] Chabrowski, J., On nonlocal problems for parabolic equations, Nagoya Math. J. 93 (1984), 109-131.

[5] Chabrowski, J., On the nonlocal problem with a functional for parabolic equation, Funkcial. Ekvac. 27 (1984), 101-123. 


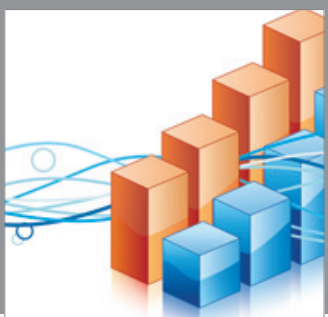

Advances in

Operations Research

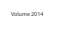

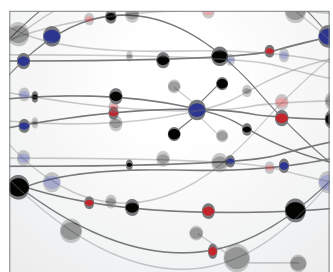

\section{The Scientific} World Journal
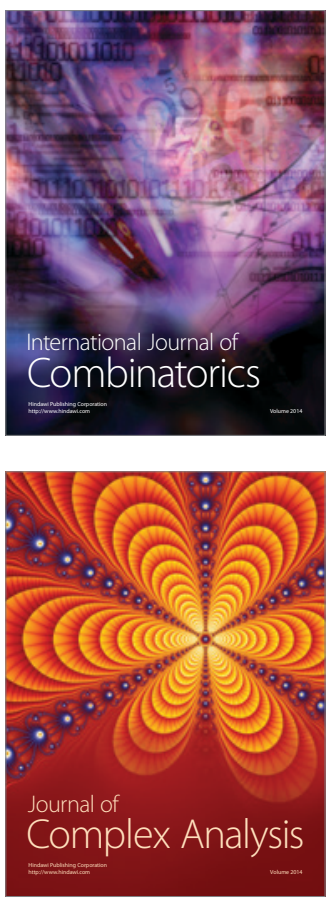

International Journal of

Mathematics and

Mathematical

Sciences
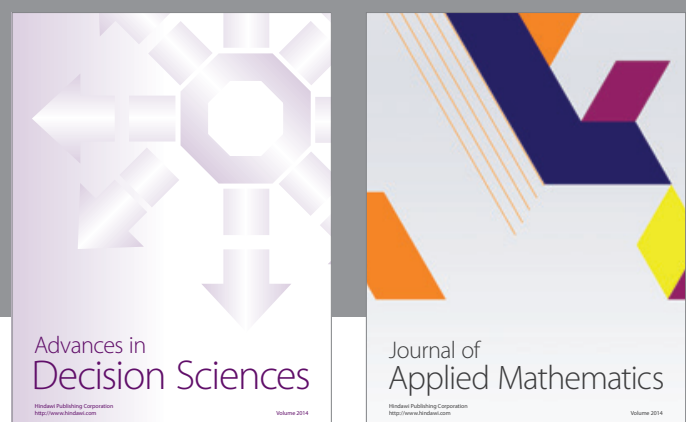

Journal of

Applied Mathematics
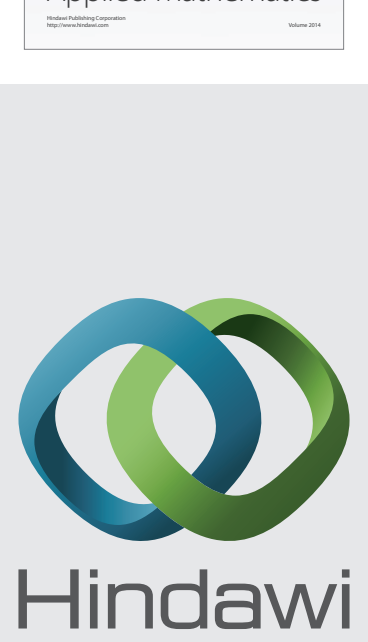

Submit your manuscripts at http://www.hindawi.com
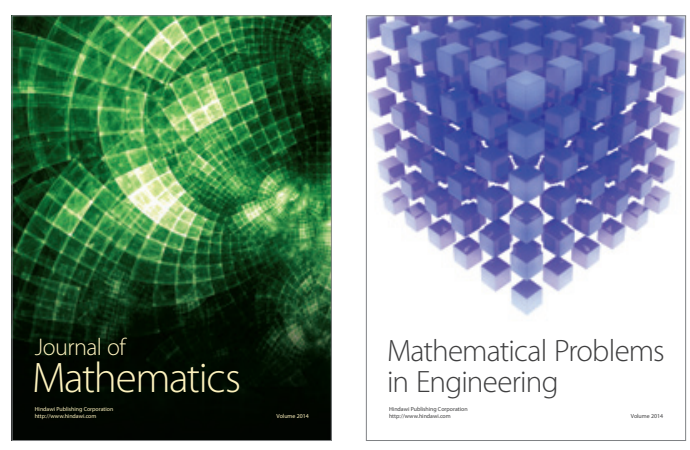

Mathematical Problems in Engineering
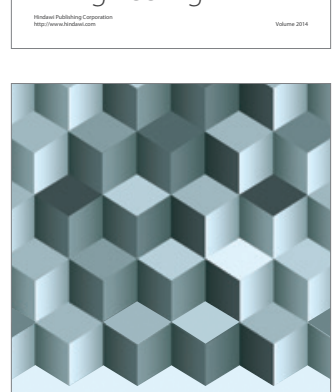

Journal of

Function Spaces
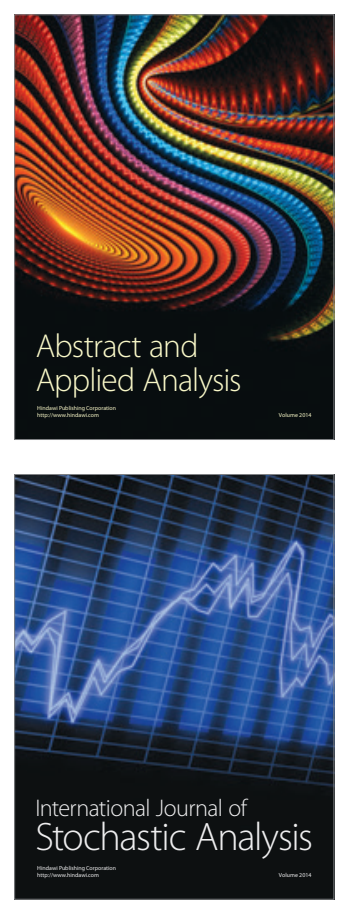

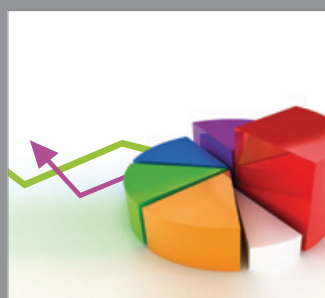

ournal of

Probability and Statistics

Promensencen
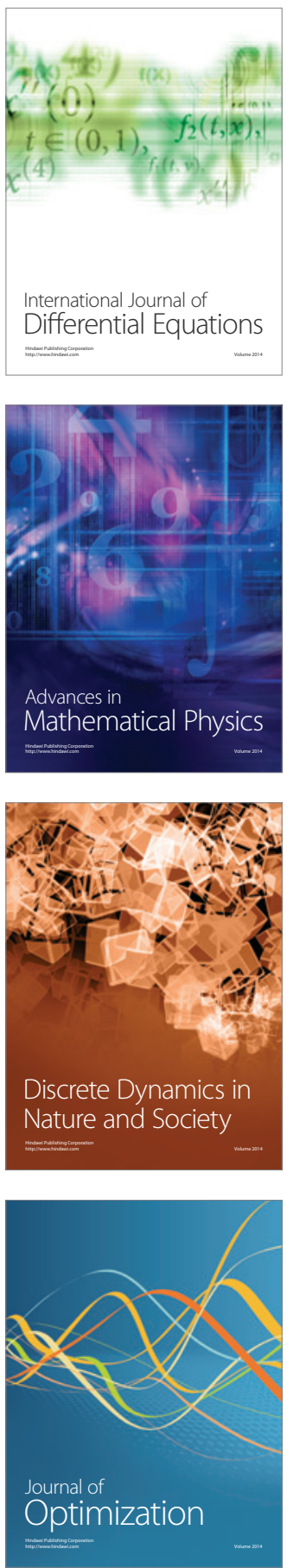